\title{
Topical applied nutraceutical antioxidant formulation reduces ocular oxidative stress
}

\author{
Peter F. Kador ${ }^{1,2}$ \\ ${ }^{1}$ Department of Pharmaceutical Sciences, College of Pharmacy and Department of \\ Ophthalmology, College of Medicine, University of Nebraska Medical Center, Omaha, Nebraska, \\ USA; ${ }^{2}$ Therapeutic Vision Inc., Omaha, Nebraska, USA
}

Corresponding Author: Peter F. Kador, PhD, FARVO, Professor, Pharmaceutical Sciences, 986025 College of Pharmacy, University of Nebraska Medical Center, Omaha, NE. and President, Therapeutic Vision, Inc., Omaha, NE, USA

Submission Date: September 7, 2016; Acceptance date: January 27, 2017; Publication date: January 31, 2017

Citation: Kador P.F. Topical applied nutraceutical antioxidant formulation reduces ocular oxidative stress. Functional Foods in Health and Disease 2017; 7(1): 17-35

\begin{abstract}
Oral nutraceutical antioxidants have shown disappointing clinical results in reducing oxidationinduced age-related cataract and other ocular diseases. Based on the hypothesis that nutraceuticals do not adequately reach the lens by oral administration, we have developed a unique topical antioxidant formulation whose active ingredients have the reported ability to reduce oxidative stress through free radical scavenging and chelating activity. This topical nutraceutical formulation was designed to mimic the in vivo activity of multifunctional antioxidants, compounds which are being developed in our laboratory to independently scavenge free radicals and selectively bind redox metals.

A comparison of the efficacy of this topical nutraceutical to multifunctional antioxidants in laboratory animal models of oxidation-induced lens changes, retinal changes, and dry eye is reviewed. Although it is less potent than the small molecule multifunctional antioxidants that will require FDA approval, the topical nutraceutical formulation beneficially reduces ocular oxidative stress. These studies suggest that this topical antioxidant may fill an unmet therapeutic need by providing a nutraceutical that beneficially reduces the effects of oxidation on age-related ocular diseases.
\end{abstract}

Keywords: oxidative stress, nutraceutical antioxidants, age-related ocular diseases, dry eye, cataracts, retinal degeneration 


\section{INTRODUCTION}

The Free Radical Theory of Aging was proposed by Denham Harman in the 1950s. This theory postulates that oxygen free radicals which are formed endogenously from normal metabolic processes play a role in aging [2]. This hypothesis sparked research which has evolved into the field of redox biology where the relationship between reactive oxygen species (ROS) and biological function(s) and disease are being identified and defined. ROS are oxygen containing reactive chemical species that are produced intracellularly as natural byproducts of normal oxygen metabolism both from inside (endogenous) and outside (exogenous) sources. Endogenous ROS plays important roles in cell signaling and homeostasis, with superoxide, hydrogen peroxide, and nitric oxide species being the most prominent. Exogenous ROS is generated from outside sources that include xenobiotics (e.g. drugs, pesticides or carcinogens), environmental pollutants (e.g. carbon based smoke), and exposure to light and other forms of radiation. The presence of free transition metals such as iron, copper, or zinc can also contribute to ROS [3]. The levels of these metals increase with aging and the presence of select diseases, in addition to from exogenous sources including the use of tobacco [3-8]. In the presence of superoxide and hydrogen peroxide radicals, transition metals such as iron or copper catalyze the generation of highly reactive hydroxyl radicals through the Fenton reaction [9].

\section{Oxidative Stress and the Eye}

ROS plays important roles in the regulation of cell survival. Moderate levels function as signals which promote cell proliferation and survival, while increased levels can induce cell death [10]. ROS production by mammalian mitochondria is especially important because it underlies oxidative damage in many pathologies and contributes to retrograde redox signaling from the organelle to the cytosol and nucleus [11]. ROS is regulated by a number of endogenous antioxidant and enzyme systems [12-14]. The breakdown of these protective antioxidant systems with age or disease results in an elevated generation of ROS, with this imbalance being defined as oxidative stress. The Fenton product induced oxidative stress; however, it cannot be readily reduced because no endogenous detoxification systems neutralize the toxic hydroxyl radicals [15]. ROS damages lipids, proteins, and DNA. Moreover, ROS has been detected in cancers and age related diseases linked to neurodegeneration, inflammation, diabetes, and vision and sensory loss. Our studies have focused on reducing ocular oxidative stress because the eye is a unique organ which is exposed to both endogenous and exogenous ROS. In the eye, endogenous ROS is associated with the high neural retinal metabolism required to convert light to electrical signals that are sent to the brain, while the exogenous ROS comes from light interacting with oxygen, both on the outer surface of the eye and from passing through the eye to the retina. This increase in endogenous and exogenous ocular ROS is also accompanied by the accumulation of select transition metals; for example, iron with age or environmental exposure to smoke, especially from tobacco [7, 16, 17].

\section{Clinical Challenges with Antioxidant Therapy}

The link between oxidative stress and age-related diseases has sparked programs designed to encourage one to acquire a healthy lifestyle which includes a diet rich in antioxidants. This has also spawned a nutraceutical industry which provides the consumer with numerous natural 
antioxidant supplements. However, the scientific evaluation of dietary changes and antioxidant supplements have been limited, while clinical evaluations have been largely inconclusive or disappointing [18-20]. This is especially true for clinical studies on the prevention of cataracts where oxidative stress has been established to play a key role in the development of age-related cataracts [21-23]. As the largest avascular tissue in the body, the lens is composed of an anterior monolayer of cuboidal lens epithelial cells (LECs) and specifically aligned fiber cells which are all enclosed in a collagenous capsule. Because it is avascular, it only receives nutrients from the aqueous humor in the anterior chamber. LECs contain mitochondria and other organelles, large amounts of cytoskeletal proteins including microtubules and actin, in addition to structural proteins called crystallins. These divide at the germinative zone and move to the equator, where they elongate and differentiate into lens fibers to form highly ordered concentric shells. Mitochondria and nuclei in these fibers cells are lost, while new crystallin proteins are produced. Differentiation occurs throughout life. Accordingly, the age of fiber cells increases with their location toward the center. At the lens nucleus, the fiber cells correspond to the fetal stage. Structurally, lens fiber cells and their proteins are highly ordered; this order must be maintained for lens clarity. The unique structural and biochemical differences between the epithelial and fiber cells result in significant differences in redox biology. These differences make the lens highly susceptible to oxidative stress, which can cause irreversible protein and membrane changes that eventually result in loss of transparency, i.e., cataract formation.

Despite the established link between oxidative stress and cataract formation, randomized, double-masked, placebo-controlled clinical trials with populations ranging from 1,000 to nearly 40,000 participants have failed to show efficacy when antioxidants or vitamin formulations were orally administered [23, 24]. Since the avascular lens receives all of its nutrients from the aqueous humor, this failure may be due to the inability of oral antioxidants to adequately cross the bloodaqueous barrier to achieve therapeutic levels in the lens. Alternatively, the antioxidant activity may not be potent enough. These possibilities are supported by results from the AREDS trials where retinal but no lens efficacy was observed [24-26]. However, retinal efficacy by the AREDS formulations is very limited.

\section{Development of Multifunctional Antioxidants.}

We have developed a new class of synthetic antioxidants called multifunctional antioxidants (MFAOs) [27-28]. These compounds use innovative therapeutic strategy, which involves multifunctional metal attenuating antioxidants targeting multiple mechanisms of radical action that are superior to compounds which only bind transition metals or scavenge free radicals. They not only scavenge free radicals but also independently sequester and redistribute free transition metals that can participate in the Fenton generation of toxic hydroxyl radicals (Figure 1). MFAOs contain a 2-amino-5-hydroxy-pyrimidine group that has 100-fold greater scavenging activity than vitamin E. They are orally active and achieve therapeutic levels in the lens in addition to the retina. In rats, MFAOs reduce cataract formation induced by whole-head gamma irradiation, as well as diabetic 
ER stress and UV light [1,29]. They also protect both the neural retina and photoreceptor layer against retinal degeneration induced by light in dark-adapted rats [30], in addition to reducing amyloid beta plaque formation in the brain of Alzheimer's transgenic mice [27]. Recent studies also suggest that these compounds protect rats from noise-induced neurodegenerative damage [31].

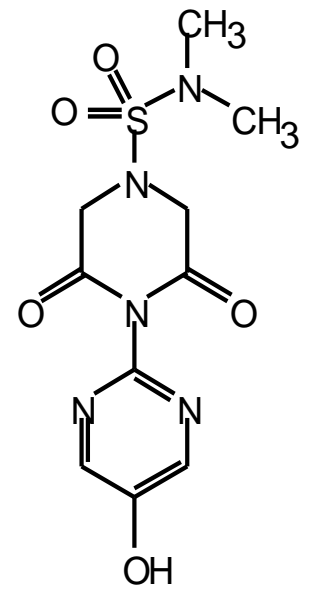

JHX-4

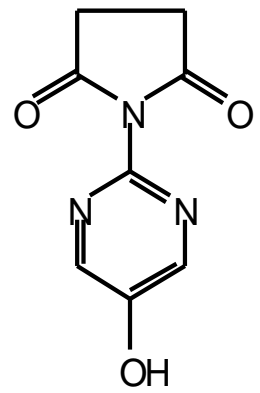

HK-2

\section{Metal Binding Radical Scavenging} Activity

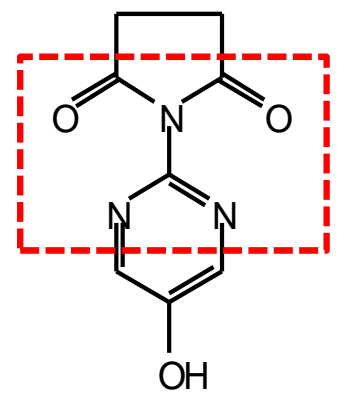

Activity

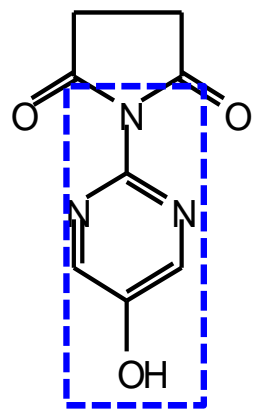

Figure 1. Representative structures of the first generation (JHX-4) and second generation (HK2) multifunctional antioxidants. The red dotted area represents atoms associated with metal binding activity while the blue dotted area represents the atoms associated with free radical scavenging activity.

\section{Topical Nutraceutical Antioxidant Formulation for Eye Health}

Since the clinical development of these agents will take years to achieve stringent FDA approval, we have investigated whether the biological activity of MFAOs in the eye can, at least in part, be mimicked by a combination of unique nutraceuticals. Based on their observed ocular antioxidant effects, we have developed and patented a nutraceutical formulation [32] (referred to here as topical nutraceutical) containing astaxanthin, resveratrol, (-)-epigallocatechin gallate (EGCG), and ethyl pyruvate (Figure 2). These were chosen for their following biological importance:

Astaxanthin is a carotenoid with 10-fold higher antioxidant activity than zeaxanthin, lutein, canthaxantin, and $\beta$-carotene. It is called "super vitamin E" because it has 100 -fold higher antioxidant activity than $\alpha$-tocopherol (vitamin E) [33]. In polar solvent, astaxanthin exists in equilibrium with its keto-enol form which possesses a hydrogen atom capable of breaking the free radical reaction in a manner similar to that of $\alpha$-tocopherol.

Resveratrol is an antioxidant present in red wine [34] that has been touted for its anti-aging properties $[35,36]$. Its antioxidant activity results from the presence of conjugated hydroxyl 
groups. Oral administration of resveratrol has limited bioavailability because of its rapid metabolism by glucuronide and sulfate conjugation in the intestine and liver [37].

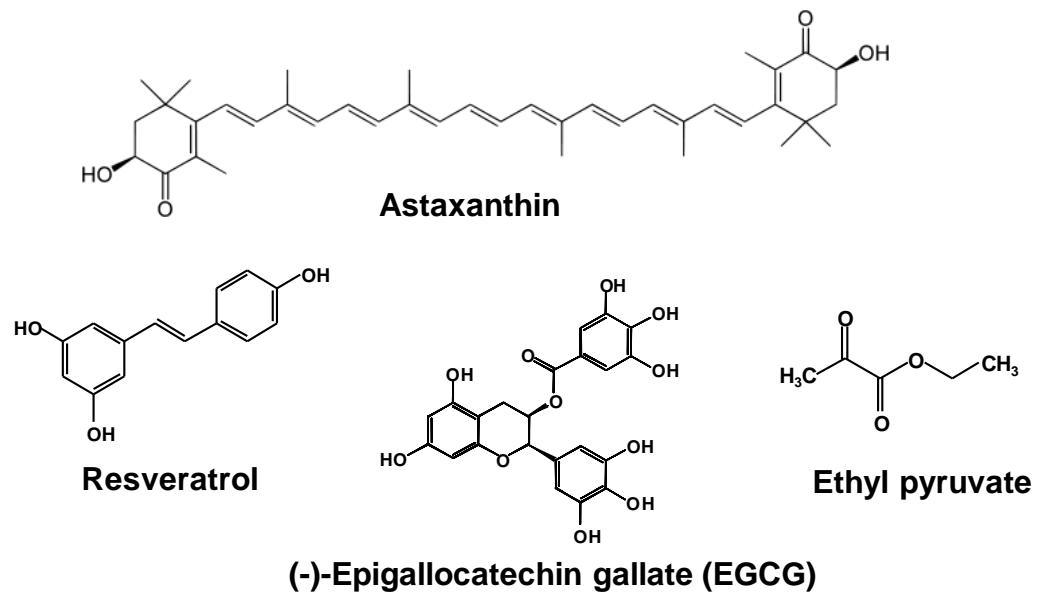

Figure 2. Structure of nutraceutical antioxidants in the topical nutraceutical formulation.

Topical application increases its bioavailability by bypassing this rapid, first pass metabolism. Long-term administration of resveratrol to mice has been reported to slow age-related degeneration and functional decline, including cataract formation [38]. In mice that are injected with LPS to induce endotoxin-induced uveitis (EIU) undergo treatment with resveratrol prior to LPS injection reduced inflammation through inhibition of oxidative damage and redox-sensitive NF- $\kappa \mathrm{B}$ activation [39]. It is also believed to ameliorate oxidative stress associated with AMD and glaucoma [40]. The addition of 50 and $100 \mathrm{mmol} / \mathrm{L}$ of resveratrol reduced the in vitro proliferation of human RPE19 cells by $10 \%$ and $25 \%$ respectively, and protected the RPE cells from hydrogen peroxide-induced cell death $[41,41]$.

EGCG, the most abundant component in green tea, has 10-fold greater antioxidant activity than vitamin E. It also has the reported ability to chelate redox reactive iron and copper [43-46]. When topically applied to the eye, it reaches the lens [47]. Studies with cultured human LECs have shown that EGCG reduces ROS associated with UVB radiation and hydrogen peroxide [46, 48-50]. It also protects human RPE cells against UVA-induced damage [51] and retina photoreceptors against oxidation induced by sodium nitroprusside [52].

Pyruvate is an endogenous antioxidant that can scavenge radicals [53-55]. When administered as the ethyl ester prodrug, it has anti-inflammatory activity and reduces organ system damage in ischemia/reperfusion injury and hemorrhagic and endotoxic shock [56]. It also beneficially effects cytokine expression and proinflammatory gene regulation [57]. When topically applied, both sodium pyruvate and its ethyl ester penetrate the cornea $[58,59]$. Both in vitro lens and in vivo diabetic rat studies report that pyruvate delayed sugar cataract formation by decreasing lens sorbitol levels [60], lipid peroxidation [61], and promoting the reduction of pyruvate to lactate [62]. The i.p. administration of pyruvate also reduces selenite cataract formation in rats [63, 64]. 
These four unique nutraceutical antioxidants possess the ability to reduce oxidative stress through both free radical scavenging and chelating activity designed to mimic the promising effects of MFAOs in the eye. Together, these nutraceuticals are topically delivered in a viscous proprietary carbomer vehicle with established topical adhesion properties which have been shown to enhance the ocular uptake of select drugs [65]. The topical delivery of these nutraceuticals was chosen based on the hypothesis that oral administration of antioxidants generally fails to achieve adequate levels in the anterior segment, including the lens, and because topical delivery bypasses first pass metabolism. Using rat models associated with cataract formation, light-induced retinal degeneration, and scopolamine-induced dry eye, the therapeutic potential of the topical administration of a 3\% suspension of the nutraceutical components in this formulation have been compared to other agents. These animal studies were approved by the University of Nebraska Medical Center Institutional Animal Care and Use Committee and conformed to the Association for Research in Vision and Ophthalmology Statement for the Use of Animals in Ophthalmic and Vision Research. For the lens studies, the following were included: Ocu-GLO ${ }^{\mathrm{TM}}$ purchased from Animal Health Quest, Bellingham, WA. The capsular contents of this oral antioxidant, designed to reduce cataracts in dogs, were dissolved in Tween 80 so that administration by gavage of the Tween mixture delivered the manufacturer's suggested amount of nutraceutical formulation per weight of rat; Kinostat ${ }^{\circledR}$, a topical aldose reductase inhibitor (ARI) from Therapeutic Vision, Inc., Omaha, NE that was administered b.i.d.; Imirestat (AL1576), an ARI from Alcon Pharmaceuticals, Ft. Worth, TX, that was orally administered in standard rat chow at a concentration of $0.0125 \mathrm{wt}$ $\%$ to deliver aldose of $\sim 17 \mathrm{mg} / \mathrm{kg} /$ day; JHX-4 (4-(5-hydroxypyrimidin-2-yl)-N,N-dimethyl-3,5dioxopiperazine-1-sulfonamide, a MFAO that was synthesized as previously described ${ }^{28}$. Topical MFAO JHX-4, prepared by suspending 3\% (w/w) of compound in Optixcare Eye Lube vehicle, was administered b.i.d; oral JHX-4 was administered as $0.05 \%(\mathrm{w} / \mathrm{w})$ in standard rat chow to give a dose of $\sim 49 \mathrm{mg} / \mathrm{kg} /$ day. The dry eye studies also utilized Optixcare Eye Lube + Hyaluron from Aventix Animal Health and I-drop Vet Plus which was purchased from imed Pharma, Dollard-desOrmeaux, QC, Canada. All three were delivered b.i.d. The retinal degeneration studies utilized the topical nutraceutical b.i.d compared to JHX-4 delivered orally in chow at a dose of $\sim 49 \mathrm{mg} / \mathrm{kg} / \mathrm{day}$.

\section{Cataracts}

Worldwide, age-related cataracts are the leading cause of blindness in addition to being one of the most common causes of visual impairment in the United States [66]. Since the progression of agerelated cataracts is gradual, it has been estimated that a 10 year delay in cataract progression could reduce the need for cataract surgery by 50\% [67]. However, the development of pharmaceutical treatments for age-related cataracts has been hampered by the lack of established animal models that develop age-related cataracts over practical time periods. Therefore, the only practical way of evaluating the efficacy of potential anti-cataract agents has been to focus on biomarkers associated with experimentally induced oxidative stress. Our studies have focused on reducing the lenticular effects of endogenous and exogenous generated oxidative stress.

\section{Diabetic Cataracts}

Diabetic cataracts develop rapidly within weeks in young rats after the induction of diabetes mellitus (DM). This development is also associated with the generation of endogenous ROS [1, 
29]. Diabetic cataracts are commonly called "sugar" cataracts because the lens changes are not specific to DM but also occur with galactosemia [68]. It has been established in both diabetic and galactosemic rat lenses that the enzyme aldose reductase reduces excess glucose or galactose to the respective sugar alcohols (polyols) sorbitol or galactitol [68]. The intracellular accumulation of these polyols in lens epithelial cells (LECs) and fiber cells causes osmotic changes that alter membrane permeability and begin the cascade of biochemical changes that lead to cataract formation $[69,70]$. The requirement for polyol-induced osmotic change has been confirmed by in vitro lens culture studies and in vivo animal studies [69, 71]. In LECs, polyol-linked osmotic stress also affects the endoplasmic reticulum (ER) and the osmotic induction of ER stress leads to the subsequent generation of endogenous ROS through an unfolded protein response (UPR) [72-74]. Sugar cataracts are inhibited in diabetic and galactosemic animals by ARIs which prevent lens polyol formation (and the subsequent induction of ER stress). However, sugar cataracts can only be delayed by antioxidants because they only address the generated endogenous ROS but not the polyol-induced osmotic stress. This was experimentally confirmed in the studies described below where the effects of antioxidants and ARIs were compared. For these studies, DM was induced in young (100 g) Sprague Dawley rats by tail vein injection of $75 \mathrm{mg} / \mathrm{kg}$ of streptozotocin [1]. Next, all rats with average blood glucose levels $>300 \mathrm{mg} / \mathrm{dL}$ were randomly divided into groups. Group 1 served as untreated controls while group 2 received 1 drop of the topical ARI Kinostat ${ }^{\circledR}$ to each eye b.i.d, group 3 received ARI imirestat orally mixed in rat chow, group 4 received the MFAO JHX-4 orally mixed in rat chow, group 5 received 1 drop of the topical nutraceutical to each eye b.i.d. and group 6 orally received the antioxidant Ocu-GLO ${ }^{\mathrm{TM}}$ once daily by gavage. Lens changes were monitored weekly using a hand-held slit lamp following the dilation of each eye with $1 \%$ tropicamide ophthalmic solution. Lens changes were evaluated by portable slit lamp using a scale of 0-3 with 0 corresponding to no lens changes; 0.5 to suture accentuation; 1 to vacuole formation; 2 to cortical opacity; and 3 to mature cataract.

As summarized in Figure 3, slit lamp examinations indicated that cataract development in untreated diabetic rats rapidly progress to the cortical or mature stage within 7 weeks of the induction of DM. This cataract formation was inhibited by both topical administration of the ARI Kinostat $^{\circledR}$ or oral ARI imirestat $[65,75]$. In contrast, oral administration of the MFAO JHX-4 or the oral anti-cataract antioxidant/vitamin formulation Ocu-GLO ${ }^{\mathrm{TM}}$ only slightly delayed the progression of cataract, with lens changes ranging from cortical to mature cataracts present at the end of the study period. This delay in cataract formation was much greater in rats receiving the topical nutraceutical with primarily suture accentuation, the initial stage of sugar cataract development that occurs before the vacuole formation only present. These slit lamp observations were subsequently confirmed at study completion by microscopic examination of the dissected lenses, where no lens changes were evident in the ARI treated rats. Additionally, measurement of glycosylated hemoglobin levels (HbA1c, Bayer Metrika A1cNOW Plus System test kit, San Diego, CA) indicated that all groups were similarly hyperglycemic over the course of this study, 
thereby removing the possibility that these observed differences in sugar cataract formation were linked to differences in the severity of diabetes.

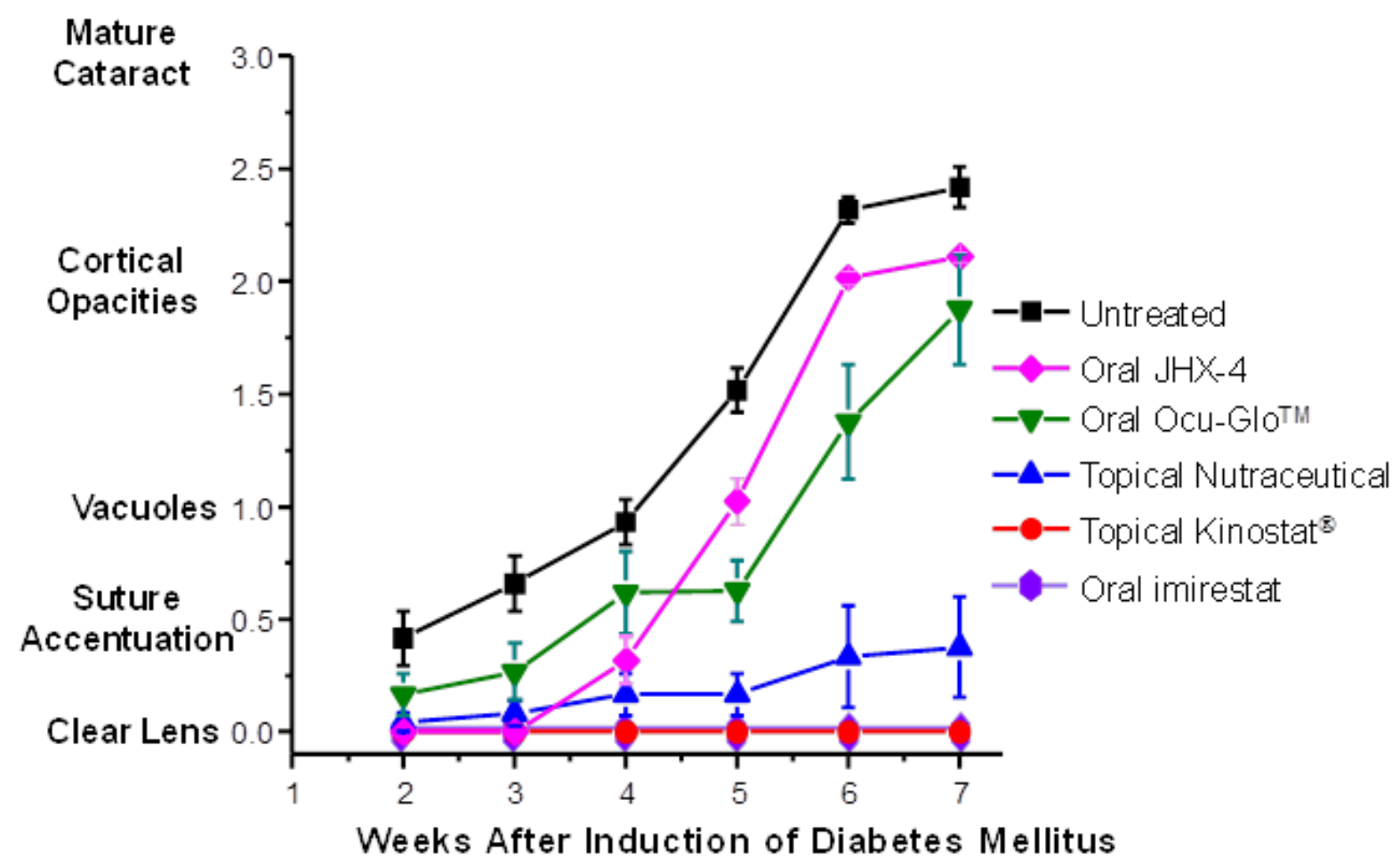

Figure 3 Effect of ARIs, topical nutraceutical, and oral MFAO JHX-4 and Ocu-GLO ${ }^{\mathrm{TM}}$ on the progression of sugar cataract formation. All agents were administered from the onset of diabetes. Oral JHX-4 and imirestat included in the graph were normalized against untreated diabetic rats from a separate study. ${ }^{1}$ The control was not statistical different $(p<0.05)$ from either oral JHX-4 or oral Ocu-Glo ${ }^{\mathrm{TM}}$. The Topical Nutraceutical was significantly $(\mathrm{p}<0.05)$ different from both the control and topical Kinostat ${ }^{\circledR}$ or oral imirestat. Values represent mean $\pm \mathrm{SEM} ; \mathrm{n}=5$ rats.

\section{UV Induced Cataracts}

Our eyes are constantly exposed to UV irradiation from sunlight and artificial lighting. Moreover, excess exposure to UV irradiation is a major risk factor for cataract and macular degeneration [76]. UV light damages the lens through the generation of exogenous ROS that decreases lens reduced glutathione (GSH) levels and leads to lens opacification. In young albino rats exposed to UV irradiation, this decrease in GSH levels occurs within 2-3 days of UV exposure [77, 78]. To investigate the efficacy of antioxidants in preventing this reduction of GSH levels, young albino rats were anesthetized and one eye was patched to serve as a dark control. The contralateral eye was then exposed to $1600 \mu \mathrm{w} / \mathrm{cm}^{2}$ of UV light (280-360 nm, UV $\mathrm{max}_{\max } 306 \mathrm{~nm}$ ) for 15 minutes which resulted in a significant decrease in lens GSH levels within 2 days after exposure. A similar 
significant decrease was also observed in Ocu-GLO ${ }^{\mathrm{TM}}$ rats where oral treatment was initiated 3 days prior to UV exposure. In contrast, similar exposure of rats treated with either $3 \% \mathrm{MFAO}$ JHX-4 or topical nutraceutical b.i.d. beginning 3 days before UV exposure protected against a significant loss of lens GSH levels. (Figure 4A).

To confirm that the reduction of GSH levels was linked to ROS generated oxidation in the lens, the lenticular levels of oxidized 4-hydroxynonenol (4-HNE) were also examined using an OxiSelect HNE Adduct Competitive ELISA Assay Kit (Cell Biolabs, Inc., San Diego, CA). As shown in Figure 4B, lens levels of 4-HNE increased with UV exposure and these levels were reduced by all antioxidant treatments. Compared to the 4-HNE levels in lenses from untreated rats exposed to UV irradiation, 4-HNE levels were only significantly reduced in rats treated with topical JHX-4 $(\mathrm{p}<0.01)$ and topical nutraceutical $(\mathrm{p}<0.05)$, while the 4-HNE levels in the oral Ocu-

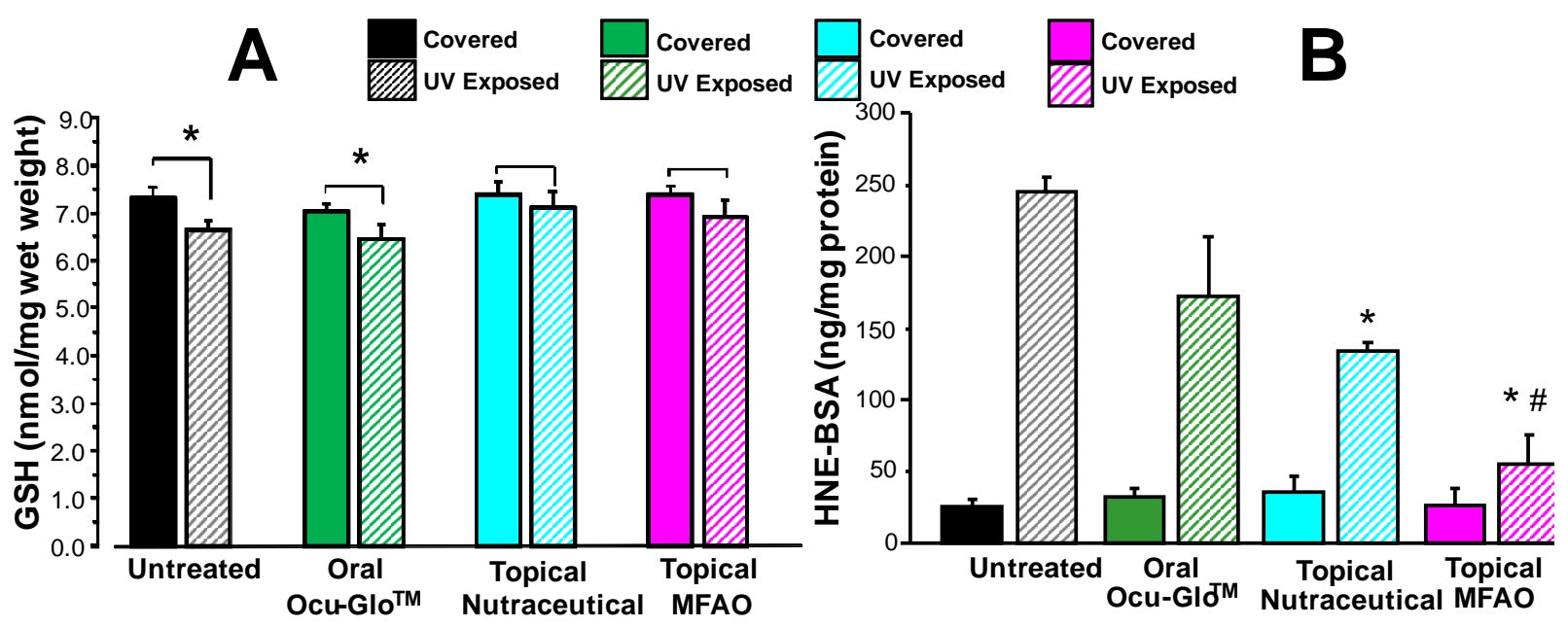

Figure 4 A. Reduction of lens GSH levels in young Sprague Dawley rats exposed to UV irradiation while their contralateral eye was covered and served as the non-exposed control. Compared to their covered contralateral eyes, significantly lower GSH levels were observed 2 days after UV exposure in lenses for untreated and oral antioxidant/vitamin formulation Ocu$\mathrm{GLO}^{\mathrm{TM}}$ treated rats, while no significant decrease was observed in rats treated with either topical MFAO JHX-4 or topical nutraceutical. B. 4-Hydroxynonenol (4-HNE) levels in the rat lenses directly correlated with the decrease in lens GSH levels in A. Lens 4-HNE levels measured 2 days after exposure to UV irradiation were reduced by antioxidant treatments in the order: topical $3 \% \mathrm{JHX}-4>$ topical nutraceutical $>$ oral Ocu-GLO ${ }^{\mathrm{TM}}>$ untreated. With the exception of topical JHX-4 treated rats, all rats had significantly higher $(\mathrm{p}<0.01)$ levels of 4-HNE in the UV exposed lenses compared to the levels in the contralateral non-irradiated control lenses. Mean \pm SEM; $n$ $=5-6$ rats. An asterisk $(*)$ denotes a significant difference in $\mathbf{A}(\mathrm{p}<0.05)$ in $\mathbf{B}(\mathrm{p}<0.01)$ compared to untreated irradiated lenses; the hatched symbol (\#) denotes a significant difference $(\mathrm{p}<0.01)$ compared to irradiated Ocu-GLO ${ }^{\mathrm{TM}}$ treated lenses.

$\mathrm{GLO}^{\mathrm{TM}}$ treated rats were not significantly reduced. However, when comparing 4-HNE levels in the exposed and unexposed contralateral eyes, the 4-HNE lens levels were statistically similar only in the MFAO JHX-4 treated rats, indicating that the protective antioxidant effects of the MFAO is slightly better compared to the topical nutraceutical. In preliminary studies, a similar 
beneficial protection was also observed when JHX-4 was orally administered 3-6 days prior to UV exposure.

\section{Light-Induced Retinal Degeneration}

When dark adapted rats are exposed to white light, ROS is generated in the neural retina along with iron dysregulation and the release of iron from ferritin $[79,80]$. This has been proposed to be an experimental animal model for dry AMD [81], and MFAOs have been shown by biochemistry, electrophysiology and histology to beneficially protect the neural retinas of these rats [30]. Since the topical nutraceutical was designed as a nutraceutical MFAO mimic, the topical nutraceutical was also evaluated in this animal model despite the fact that topically administered drugs are known, in general, to have only minimal effects on the retina.

For these studies, Wistar rats were orally treated with either diet containing $0.05 \%$ of the MFAO JHX-4 or topically treated b.i.d. with topical nutraceutical from the onset of two-week dark adaption. Each rat was then individually exposed for 3 hours to 1000 lx of cool white fluorescent light (Lights of America, Los Angeles, CA). An additional group of non-treated rats were also placed into the light box apparatus for three hours, but not exposed to light (non-light-damaged rats, NLD). Immediately following light exposure, a portion of light exposed rats from each group and non-light exposed rats were immediately euthanized, the eyes enucleated, and the neural retinas carefully dissected and frozen on dry ice. The remaining rats in each group were then returned to the dark environment. Following 5-7 days of dark recovery from intense light exposure, the retinal neural functions of the remaining rats in each group along with their corresponding non-light-damaged controls were evaluated by non-invasive electroretinography (ERG) using a UTAS system (LKC Technologies Inc., Gaithersburg, MD) [30]. Finally, all rats were euthanized seven days after light exposure by carbon dioxide asphyxiation and the dorsal ventral orientation of each eye was marked with a cautery tool. The eyes were then enucleated and fixed in a 3:1 (v/v) methanol: acetic acid solution, before being processed for subsequent morphological analyses.

Exposure to light resulted in an increase of oxidative stress markers that include 4-HNE adducts formed with histidine following the non-enzymatic oxidation of polyunsaturated fatty acids and nitrotyrosine-modified proteins resulting from tyrosine residues being oxidized by peroxynitrite formed by the reaction of nitric oxide with superoxide anions. As shown in Figure 5, both the 4hydroxynonenal (HNE)-histidine adduct levels (A) and nitrotyrosine adduct levels (B) determined by ELISA (Cell Biolabs, Inc., San Diego, CA), increased in the neural retinas of untreated rats exposed to light after 2-weeks of dark adaptation. Neither oxidative marker significantly increased in similar light exposed rats treated with the MFAO JHX-4 (Figure 5 A, B). Significantly less increases were also observed in the topical nutraceutical treated rats. ROS and free radicals generated by retinal exposure to acute excessive light were also mediated by the thioredoxin (TRx) / thioredoxin reductase (TRxR) defense system [82]. Therefore, the expression levels of both TRx and TRxR in the neural retinas of rats were examined by western blot using commercially available antibodies against TRx (Cell Signaling Technology, Danvers, MA) and TRxR (Abcam, Inc., Cambridge, MA). The expression of both increased in untreated rats exposed to light (Figure 5C, D) and treatment with either JHX-4 or topical nutraceutical significantly lowered the expression 
of TRxR in the light exposed neural retinas. However, only the MFAO JHX-4 reduced the expression of TRx.

A

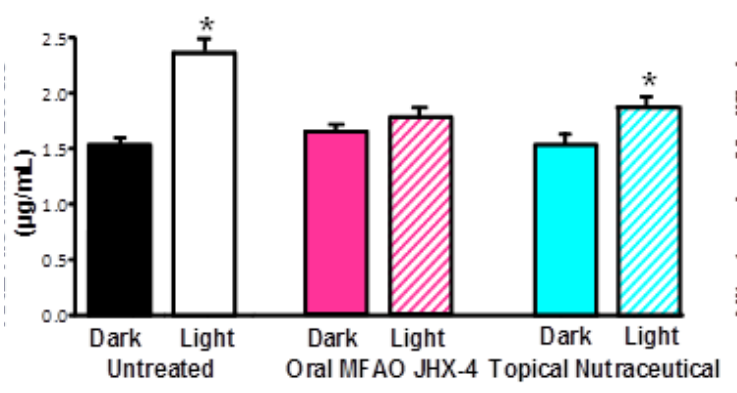

C

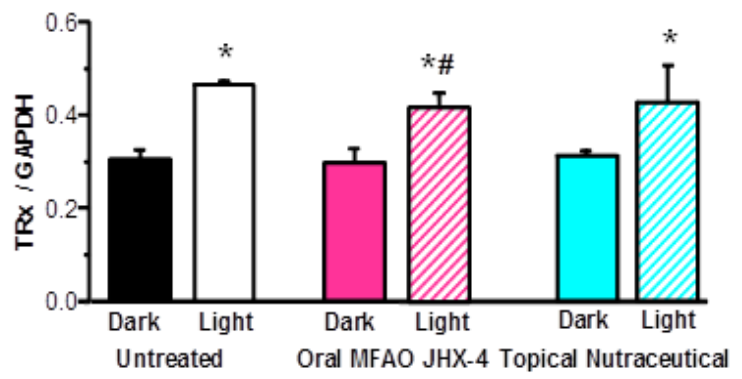

B
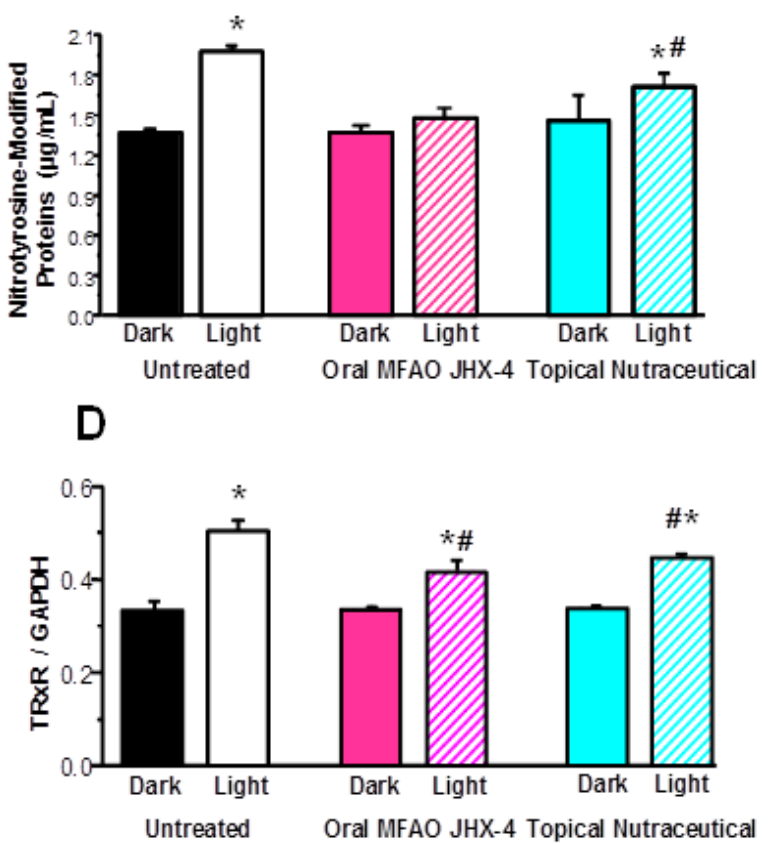

Figure 5 Effects of the oral MFAO JHX-4 and topical nutraceutical on biomarkers of light induced oxidative stress in the neural retinas of rats. A compares the levels of 4hydroxynonenal (HNE)-histidine adduct while $\mathbf{B}$ shows the nitrotyrosine adduct levels.

Both the adduct and expression levels were increased in light exposed untreated rats and reduced by antioxidant treatments. Mean \pm S.D.; $n=6$. An asterisk (*) denotes a significant difference $(\mathrm{p}<0.05)$ when compared to the untreated, non-light-damaged group while the hatched symbol $(\#)$ denotes a significant difference $(\mathrm{p}<0.05)$ compared to the untreated light damaged group.

Histological measurements confirmed the reduction of retinal oxidative stress. Intense light exposure results in photoreceptors thinning in the outer nuclear layer (ONL), with damage more pronounced in the superior compared to inferior hemisphere (Figure 6A, B). The photoreceptor layer in light exposed rats treated with JHX-4 showed decreases of $4 \%$ in the inferior hemisphere and $21 \%$ in the superior hemisphere (Figure 6C). Only a slight decrease in both the inferior hemisphere and superior hemisphere showed when compared to dark adapted rats that were not exposed to light. In contrast, the photoreceptor layer was only slightly protected in the Optixcare $\mathrm{EH}^{\mathrm{TM}}$ treated rats (Figure 6D).

This difference in photoreceptor thickness protection was more apparent by ERG measurements, a procedure that measures the function of photoreceptors (rods and cones), inner retinal cells (bipolar and amacrine cells), and ganglion cells through the measurement of electrical responses [83-85]. The a-wave is derived from the rods and cones of the outer photoreceptor layers while the b-wave is derived from the inner retina, predominantly Muller and ON-bipolar cells. This b-wave is the most common component of the ERG used in clinical and experimental analysis of retinal function. Photopic measurements are generally a cone response while rod function is 
present in the scotopic measurements. In the untreated light exposed rats, the average maximal scotopic a-wave and $b$-wave amplitudes were significantly $(\mathrm{p}<0.05)$ reduced by $30 \%$ and $25 \%$ respectively, with the maximum amplitude being significantly reduced by $25 \%$ in the photopic ERG b-wave response. None of these reductions were observed in similar light exposed rats treated with the MFAO JHX-4. In contrast, no ERG protection was observed with the topical nutraceutical.
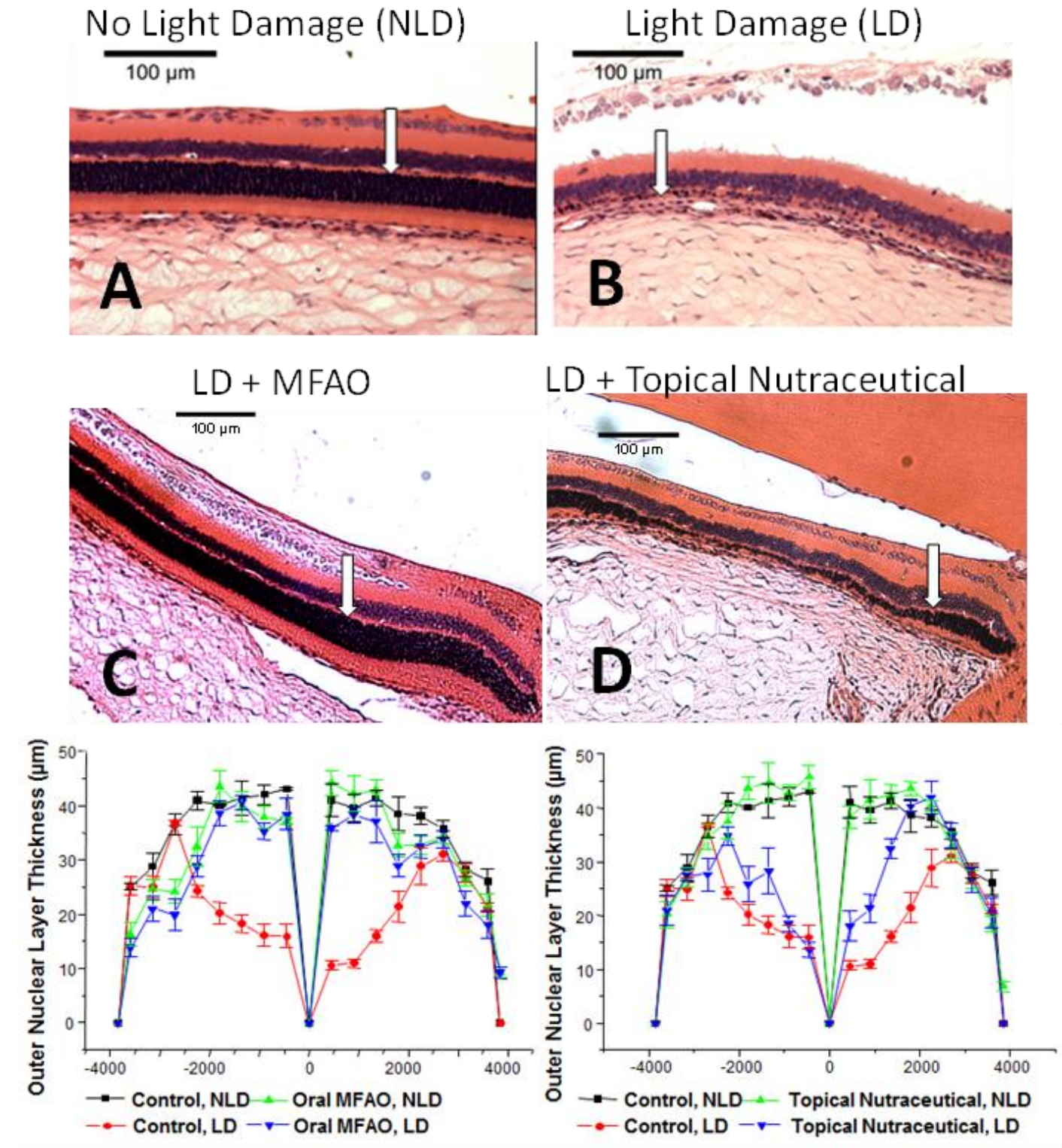

Figure 6 Light-induced destruction of the retinal outer nuclear layer (ONL) can be reduced by the presence of antioxidants. Photomicrographs of the superior hemisphere illustrate photoreceptor thinning in ONL (arrow). The NL thickness of non-light damaged and light damaged retinas treated with or without the multifunctional MFAO JHX-4 or the topical nutraceutical are below their respective photomicrograph. The MFAO had superior protection. $\mathrm{n}=3$. Mean \pm S.E.M. 


\section{Keratoconjunctivitis Sicca (KCS)}

Keratoconjunctivitis sicca (KCS), commonly called dry eye, occurs in nearly five million Americans 50 years of age and older [86]. Although more frequently experienced in the elderly, KCS can occur at any age with symptoms that include dryness/itching, burning/stinging, watering or a gritty or sandy sensation. It can also include a sensitivity to light, blurry vision, or difficulty with night vision. These symptoms can be temporary or can increase to become chronic. KCS results from either lacrimal glands that fail to produce enough of the watery component of tears to maintain a healthy eye surface, or inflammation due to the meibomian gland function, which makes the lipid or oily part of tears that slows tear evaporation and keeps the tears stable. The first line of therapy for KCS is the use of "comfort agents" that include non-prescription (OTC) artificial tears, gels, gel inserts, and ointments.

They offer temporary relief and provide an important replacement of naturally produced tears in patients with aqueous tear deficiency. Subsequently, as KCS is also linked to oxidative stress and inflammation, prescription anti-inflammatory, or immunosuppressant medications including cyclosporin or temporary corticosteroids may be used to decrease corneal damage, increase basic tear production, and reduce the symptoms of dry eye. Nutraceuticals including combined antioxidants and omega-3 essential fatty acid formulations [87] or topical epigallocatechin gallate [88] and castor oil have also been used.

Optixcare $^{\mathrm{TM}}$ carbomer gel and carbomer gel containing hyaluronic acid formulations are comfort agents used in the veterinary market for alleviating the clinical symptoms of dry eye. Since the ingredients in our topical nutraceutical formulation have been reported to demonstrate not only antioxidant but also anti-inflammatory activity, studies comparing the effects of the topical nutraceutical to carbomer comfort agents were compared. In these studies, KCS was induced in adult female Lewis rats by twice-daily subcutaneous injection of a $0.1 \mathrm{~mL}$ aqueous solution containing $24 \mathrm{mg}$ of scopolamine (48 $\mathrm{mg}$ total dosage per day) [89]. The tear flow in these rats was measured weekly prior using Zone-Quick ${ }^{\mathrm{TM}}$ Phenol Red Threads (FCI Ophthalmics, Pembroke, Massachusetts) by anesthetizing each rat with isoflurane. The Threads were place in the palpebral conjunctiva of the open eye for 15 seconds and then immediately removed and measured. In each rat, tear flow was determined as the average of both eyes.

As shown in Figure 7 continued injection of scopolamine resulted in decreased tear flow in untreated rats. A similar decrease in tear flow was observed in rats topically receiving b.i.d the comfort agents Optixcare $^{\mathrm{TM}}$, Optixcare ${ }^{\mathrm{TM}}$ with hyaluronic acid (HA), or i-Drop hyaluronic acid. In contrast, the tear flow was maintained in rats receiving topical nutraceutical b.i.d. This suggests that the antioxidant/anti-inflammatory activity of the ingredients in the topical nutraceutical are beneficial for treating KCS. 


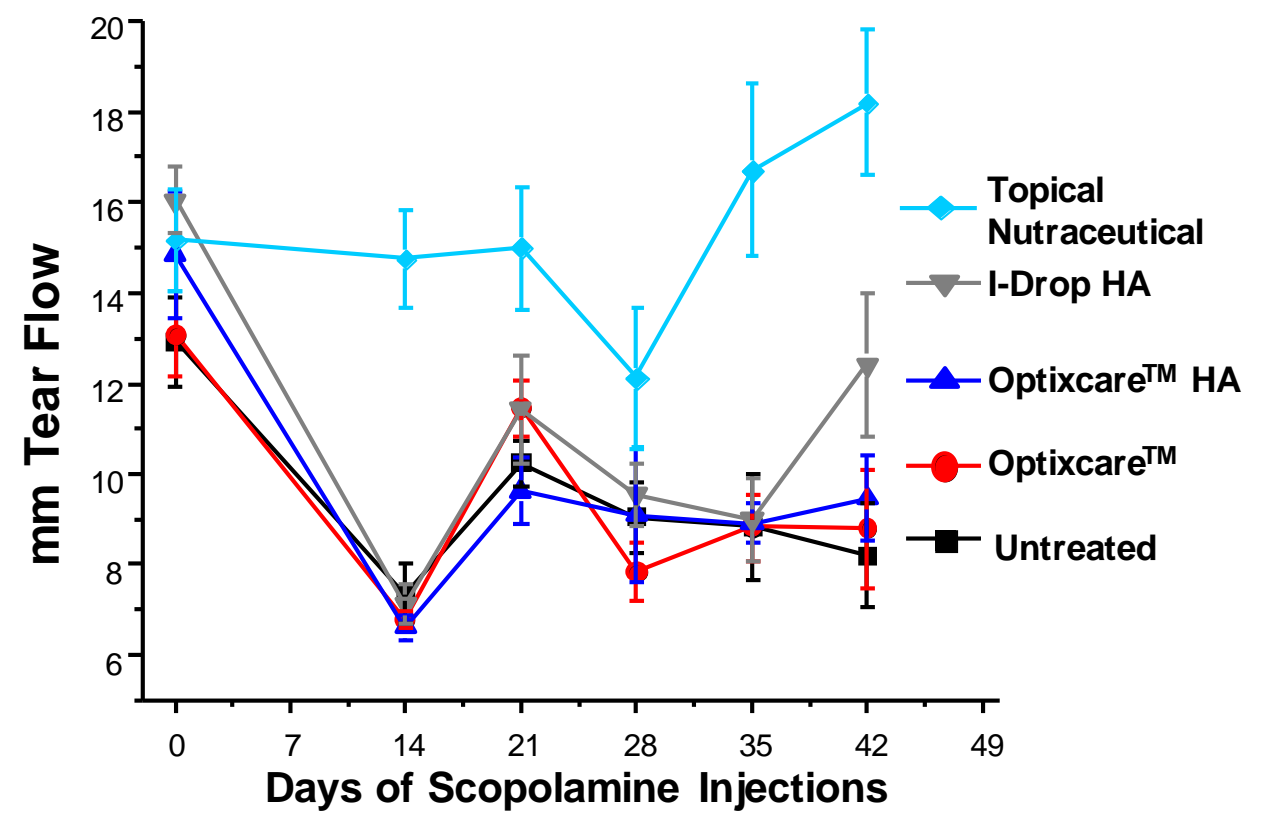

Figure 7 Effect of topical carbomer gels on tear flow in scopolamine injected rats. Continuous scopolamine injections resulted in decreased tear flow in both the untreated control rats and rats treated with the comfort agents Optixcare ${ }^{\mathrm{TM}}$, Optixcare $^{\mathrm{TM}}$ with hyaluronic acid (HA), and i-Drop hyaluronic acid (HA). Tear flow was maintained in similar rats treated with the topical nutraceutical. SEM; $n=6$ rats.

\section{CONCLUSION}

The results demonstrate that the topical application of an antioxidant formulation with combined free radical scavenging, transition metal binding, and anti-inflammatory activity has beneficial effects on reducing oxidative stress that is associated with age-related ocular changes. Moreover, the application this topical nutraceutical mimics to some extent the effects of MFAOs in the eye. The small beneficial effects of the topical nutraceutical on oxidation-linked retinal changes were unexpected, as agents topically applied to the eye generally do not reach the retina. Retinal antioxidant efficacy is anticipated to increase by using a combination of oral and topical administration of the formula ingredients.

For the veterinary market, this formulation has been licensed to CLC MEDICA, Waterdown, ON, Canada and is being marketed as Optixcare ${ }^{\mathrm{TM}}$ Eye Health primarily to veterinary ophthalmologists. Clinical observations to date suggest that this formulation is beneficial, not only for treating KCS but also apparently delaying age-related cataracts. During its use, an interesting new clinical indication for Optixcare ${ }^{\mathrm{TM}}$ Eye Health has come from observations by several veterinary ophthalmologists. This indication is that this topical nutraceutical with antioxidant/antiinflammatory/chelation properties reduces tear staining (epiphora) in dogs. Tear staining are reddish-brown streaks under a dog's (or cat's) eyes present in the medial canthus that are usually caused by epiphora, i.e., an overflow of tears. The color comes from porphyrins, which are naturally occurring molecules containing iron that are formed as waste products from the breakdown of red blood cells and exposure to sunlight that can darken these stains. This condition is much more prevalent in certain breeds (for example, the toy poodle, Maltese, the Lhasa Apso, and the Shih Tzu), and is much more obvious in animals with light-colored coats. While tear 
staining is generally no more than a minor annoyance, it can also be a symptom of serious eye health problems that include ingrown eyelashes, infections, unusually large tear glands, usually small tear duct openings, entropion, glaucoma, ear infections, or brachycephalic syndrome. Tear staining from excess tearing can also result from certain medications, exposure to secondhand smoke, stress, and diets. At present, a multi-centered clinical trial with Optixcare ${ }^{\mathrm{TM}}$ Eye Health is being conducted to validate this new clinical indication.

The ability to prevent KSC is also in the process of being clinically investigated in humans with studies anticipated to begin in 2016. While a topical formulation of select antioxidant with antioxidant/anti-inflammatory/chelation properties appears to be promising for reducing agerelated oxidative stress, it is anticipated that future beneficial effects will become apparent through extended clinical use as an OTC medication and established through anticipated future clinical trials.

\section{REFERENCES}

1. Randazzo J, Zhang P, Makita J, Blessing K, Kador PF. Orally active multi-functional antioxidants delay cataract formation in streptozotocin (type 1) diabetic and gammairradiated rats. PloS one 2011;6:e18980.

2. Harman DD. About "Origin and evolution of the free radical theory of aging: a brief personal history, 1954-2009". Biogerontology;10:783-.

3. Killilea DWDW. Iron accumulation during cellular senescence. Annals of the New York Academy of Sciences;1019:365-7.

4. Dunaief JL. Iron induced oxidative damage as a potential factor in age-related macular degeneration: the Cogan Lecture. Invest Ophthalmol Vis Sci 2006;47:4660-4.

5. Smith DG, Cappai R, Barnham KJ. The redox chemistry of the Alzheimer's disease amyloid beta peptide. Biochimica et biophysica acta 2007;1768:1976-90.

6. Brewer GJ. Iron and copper toxicity in diseases of aging, particularly atherosclerosis and Alzheimer's disease. Exp Biol Med (Maywood) 2007;232:323-35.

7. Avunduk AM, Yardimci S, Avunduk MC, Kurnaz L, Kockar MC. Determinations of some trace and heavy metals in rat lenses after tobacco smoke exposure and their relationships to lens injury. Exp Eye Res 1997;65:417-23.

8. Lois N, Abdelkader E, Reglitz K, Garden C, Ayres JG. Environmental tobacco smoke exposure and eye disease. Br J Ophthalmol 2008;92:1304-10.

9. Wardman P, Candeias LP. Fenton chemistry: an introduction. Radiat Res 1996;145:523-31.

10. Trachootham DD. Redox regulation of cell survival. Antioxidants \& redox signaling;10:1343-74.

11. Murphy MP. How mitochondria produce reactive oxygen species. Biochem J 2009;417:1 13.

12. Camhi SLS. The oxidative stress response. New horizons (Baltimore, Md);3:170-82.

13. Ray PD, Huang BW, Tsuji Y. Reactive oxygen species (ROS) homeostasis and redox regulation in cellular signaling. Cell Signal 2012;24:981-90.

14. Rizzo AM, Berselli P, Zava S, et al. Endogenous antioxidants and radical scavengers. Adv Exp Med Biol 2010;698:52-67. 
15. Ramana BV, Raju TN, Kumar VV, Reddy PU. Defensive role of quercetin against imbalances of calcium, sodium, and potassium in galactosemic cataract. Biol Trace Elem Res 2007;119:35-41.

16. Janik-Papis K, Ulinska M, Krzyzanowska A, et al. [Role of oxidative mechanisms in the pathogenesis of age-related macular degeneration]. Klin Oczna 2009;111:168-73.

17. Avunduk AM, Yardimci S, Avunduk MC, Kurnaz L. Cataractous changes in rat lens following cigarette smoke exposure is prevented by parenteral deferoxamine therapy. Arch Ophthalmol 1999;117:1368-72.

18. Joshi SS. Vitamin Supplementation in the Elderly. Clinics in geriatric medicine;31:355-66.

19. Moser MA, Chun OK. Vitamin C and Heart Health: A Review Based on Findings from Epidemiologic Studies. Int J Mol Sci 2016;17.

20. Ozben TT. Antioxidant supplementation on cancer risk and during cancer therapy: an update. Current topics in medicinal chemistry 2015;15:170-8.

21. Thiagarajan R, Manikandan R. Antioxidants and cataract. Free radical research 2013;47:33745.

22. Michael R, Bron AJ. The ageing lens and cataract: a model of normal and pathological ageing. Philos Trans R Soc Lond B Biol Sci 2011;366:1278-92.

23. Chiu CJ, Taylor A. Nutritional antioxidants and age-related cataract and maculopathy. Experimental eye research 2007;84:229-45.

24. Chew EY. Nutrition effects on ocular diseases in the aging eye. Investigative ophthalmology \& visual science 2013;54:ORSF42-7.

25. Chiu CJ, Milton RC, Klein R, Gensler G, Taylor A. Dietary compound score and risk of agerelated macular degeneration in the age-related eye disease study. Ophthalmology 2009;116:939-46.

26. Lawrenson JGJG. Controversies in the Use of Nutritional Supplements in Ophthalmology. Current pharmaceutical design 2015;21:4667-72.

27. Kawada H, Blessing K, Kiyota T, Woolman T, Winchester L, Kador PF. Effects of multifunctional antioxidants on mitochondrial dysfunction and amyloid-beta metal dyshomeostasis. J Alzheimers Dis 2015;44:297-307.

28. Jin H, Randazzo J, Zhang P, Kador PF. Multifunctional antioxidants for the treatment of agerelated diseases. J Med Chem 2010;53:1117-27.

29. Kador PF, Guo C, Kawada H, Randazzo J, Blessing K. Topical nutraceutical Optixcare EH ameliorates experimental ocular oxidative stress in rats. J Ocul Pharmacol Ther 2014;30:593602.

30. Randazzo J, Zhang Z, Hoff $\mathrm{M}$, et al. Orally active multi-functional antioxidants are neuroprotective in a rat model of light-induced retinal damage. PloS one 2011;6:e21926.

31. Salvi R, Kador, P, Chen, G.-D.,Ding, D., Jiang, H., Manohar, S., Tseng, J., Daszynski, D.D., Woolman, T. . Protection Against Noise-Induced Hearing Loss by HK-2, a Novel Multifunctional Antioxidant. Association for Research in Otolaryngology 40th MidWinter Meeting. Baltimore, MD2017.

32. Kador PF, inventor Antioxidant eye drops USA patent 9,173,915. 2015 November 3, 2015.

33. Higuera-Ciapara I, Felix-Valenzuela L, Goycoolea FM. Astaxanthin: a review of its chemistry and applications. Crit Rev Food Sci Nutr 2006;46:185-96. 
34. Pervaiz S. Resveratrol--from the bottle to the bedside? Leuk Lymphoma 2001;40:491-8.

35. Rocha-Gonzalez HI, Ambriz-Tututi M, Granados-Soto V. Resveratrol: a natural compound with pharmacological potential in neurodegenerative diseases. CNS Neurosci Ther 2008;14:234-47.

36. Anekonda TS. Resveratrol--a boon for treating Alzheimer's disease? Brain Res Rev 2006;52:316-26.

37. Walle T, Hsieh F, DeLegge MH, Oatis JE, Jr., Walle UK. High absorption but very low bioavailability of oral resveratrol in humans. Drug Metab Dispos 2004;32:1377-82.

38. Pearson KJ, Baur JA, Lewis KN, et al. Resveratrol delays age-related deterioration and mimics transcriptional aspects of dietary restriction without extending life span. Cell Metab 2008;8:157-68.

39. Kubota S, Kurihara T, Mochimaru H, et al. Prevention of ocular inflammation in endotoxininduced uveitis with resveratrol by inhibiting oxidative damage and nuclear factor-kappaB activation. Invest Ophthalmol Vis Sci 2009;50:3512-9.

40. Rhone M, Basu A. Phytochemicals and age-related eye diseases. Nutr Rev 2008;66:465-72.

41. King RE, Kent KD, Bomser JA. Resveratrol reduces oxidation and proliferation of human retinal pigment epithelial cells via extracellular signal-regulated kinase inhibition. Chem Biol Interact 2005;151:143-9.

42. Sheu SJ, Bee YS, Chen $\mathrm{CH}$. Resveratrol and large-conductance calcium-activated potassium channels in the protection of human retinal pigment epithelial cells. J Ocul Pharmacol Ther 2008;24:551-5.

43. Hanneken A, Lin FF, Johnson J, Maher P. Flavonoids protect human retinal pigment epithelial cells from oxidative-stress-induced death. Invest Ophthalmol Vis Sci 2006;47:3164-77.

44. Mandel SA, Amit T, Kalfon L, Reznichenko L, Weinreb O, Youdim MB. Cell signaling pathways and iron chelation in the neurorestorative activity of green tea polyphenols: special reference to epigallocatechin gallate (EGCG). J Alzheimers Dis 2008;15:211-22.

45. Reznichenko L, Amit T, Zheng H, et al. Reduction of iron-regulated amyloid precursor protein and beta-amyloid peptide by (-)-epigallocatechin-3-gallate in cell cultures: implications for iron chelation in Alzheimer's disease. J Neurochem 2006;97:527-36.

46. Yao K, Ye P, Zhang L, Tan J, Tang X, Zhang Y. Epigallocatechin gallate protects against oxidative stress-induced mitochondria-dependent apoptosis in human lens epithelial cells. Molecular vision 2008;14:217-23.

47. Zigman S, Rafferty NS, Rafferty KA, Lewis N. Effects of green tea polyphenols on lens photooxidative stress. Biol Bull 1999;197:285-6.

48. Yao J, Liu Y, Wang X, et al. UVB radiation induces human lens epithelial cell migration via NADPH oxidase-mediated generation of reactive oxygen species and up-regulation of matrix metalloproteinases. International journal of molecular medicine 2009;24:153-9.

49. Heo J, Lee BR, Koh JW. Protective effects of epigallocatechin gallate after UV irradiation of cultured human lens epithelial cells. Korean journal of ophthalmology : KJO 2008;22:1836. 
50. Wu ZH, Wang MR, Yan QC, Pu W, Zhang JS. [UV-induced DNA damage and protective effects of antioxidants on DNA damage in human lens epithelial cells studied with comet assay]. [Zhonghua yan ke za zhi] Chinese journal of ophthalmology 2006;42:1002-7.

51. Chan CM, Huang JH, Lin HH, et al. Protective effects of (-)-epigallocatechin gallate on UVA-induced damage in ARPE19 cells. Molecular vision 2008;14:2528-34.

52. Zhang B, Osborne NN. Oxidative-induced retinal degeneration is attenuated by epigallocatechin gallate. Brain research 2006;1124:176-87.

53. Varma SD, Hegde KR, Kovtun S. Attenuation and delay of diabetic cataracts by antioxidants: effectiveness of pyruvate after onset of cataract. Ophthalmologica 2005;219:309-15.

54. Hegde KR, Varma SD. Morphogenetic and apoptotic changes in diabetic cataract: prevention by pyruvate. Mol Cell Biochem 2004;262:233-7.

55. Hegde KR, Varma SD. Prevention of cataract by pyruvate in experimentally diabetic mice. Mol Cell Biochem 2005;269:115-20.

56. Reade MC, Fink MP. Bench-to-bedside review: Amelioration of acute renal impairment using ethyl pyruvate. Crit Care 2005;9:556-60.

57. Das UN. Pyruvate is an endogenous anti-inflammatory and anti-oxidant molecule. Med Sci Monit 2006;12:RA79-84.

58. Chandra P, Hegde KR, Varma SD. Possibility of topical antioxidant treatment of cataracts: corneal penetration of pyruvate in humans. Ophthalmologica 2009;223:136-8.

59. Hegde KR, Kovtun S, Varma SD. Intraocular penetration of pyruvate following its topical administration in mice. Mol Cell Biochem 2009.

60. Cheng HM, Cheng FY, Tanaka GH, Xiong J, Pfleiderer B. Manipulating rat lens glucose metabolism with exogenous substrates. Exp Eye Res 1995;61:479-86.

61. Zhao W, Devamanoharan PS, Henein M, Ali AH, Varma SD. Diabetes-induced biochemical changes in rat lens: attenuation of cataractogenesis by pyruvate. Diabetes Obes Metab 2000;2:165-74.

62. Beyer-Mears A, Diecke FP, Mistry K, Ellison C, Cruz E. Effect of pyruvate on lens myoinositol transport and polyol formation in diabetic cataract. Pharmacology 1997;55:78-86.

63. Varma SD, Devamanoharan PS, Morris SM. Prevention of cataracts by nutritional and metabolic antioxidants. Crit Rev Food Sci Nutr 1995;35:111-29.

64. Varma SD, Ramachandran S, Devamanoharan PS, Morris SM, Ali AH. Prevention of oxidative damage to rat lens by pyruvate in vitro: possible attenuation in vivo. Curr Eye Res 1995;14:643-9.

65. Kador PF, Webb TR, Bras D, Ketring K, Wyman M. Topical KINOSTAT ameliorates the clinical development and progression of cataracts in dogs with diabetes mellitus. Veterinary ophthalmology 2010;13:363-8.

66. Iroku-Malize T, Kirsch S. Eye Conditions in Older Adults: Cataracts. FP Essent 2016;445:17-23.

67. Kupfer C. Bowman lecture. The conquest of cataract: a global challenge. Trans Ophthalmol Soc U K 1985;104 ( Pt 1):1-10.

68. Kinoshita JH. Mechanisms initiating cataract formation. Proctor Lecture. Invest Ophthalmol 1974;13:713-24. 
69. Kador PF, Wyman M, Oates PJ. Aldose reductase, ocular diabetic complications and the development of topical Kinostat((R)). Prog Retin Eye Res 2016;54:1-29.

70. Kinoshita JH, Kador P, Catiles M. Aldose reductase in diabetic cataracts. JAMA 1981;246:257-61.

71. Zhang P, Xing K, Randazzo J, Blessing K, Lou MF, Kador PF. Osmotic stress, not aldose reductase activity, directly induces growth factors and MAPK signaling changes during sugar cataract formation. Experimental eye research 2012;101:36-43.

72. Ikesugi K, Mulhern ML, Madson CJ, et al. Induction of endoplasmic reticulum stress in retinal pericytes by glucose deprivation. Curr Eye Res 2006;31:947-53.

73. Mulhern ML, Madson CJ, Danford A, Ikesugi K, Kador PF, Shinohara T. The unfolded protein response in lens epithelial cells from galactosemic rat lenses. Invest Ophthalmol Vis Sci 2006;47:3951-9.

74. Mulhern ML, Madson CJ, Kador PF, Randazzo J, Shinohara T. Cellular osmolytes reduce lens epithelial cell death and alleviate cataract formation in galactosemic rats. Mol Vis 2007;13:1397-405.

75. Kador PF, Randazzo J, Babb T, et al. Topical aldose reductase inhibitor formulations for effective lens drug delivery in a rat model for sugar cataracts. J Ocul Pharmacol Ther 2007;23:116-23.

76. Roberts JE. Ultraviolet radiation as a risk factor for cataract and macular degeneration. Eye \& contact lens 2011;37:246-9.

77. Kronschlager M, Lofgren S, Yu Z, Talebizadeh N, Varma SD, Soderberg P. Caffeine eye drops protect against UV-B cataract. Experimental eye research 2013;113:26-31.

78. Giblin FJ, Leverenz VR, Padgaonkar VA, et al. UVA light in vivo reaches the nucleus of the guinea pig lens and produces deleterious, oxidative effects. Experimental eye research 2002;75:445-58.

79. Organisciak DT, Vaughan DK. Retinal light damage: mechanisms and protection. Prog Retin Eye Res 2010;29:113-34.

80. Ohishi K, Zhang XM, Moriwaki S, Hiramitsu T, Matsugo S. Iron release analyses from ferritin by visible light irradiation. Free Radic Res 2005;39:875-82.

81. Wielgus AR, Collier RJ, Martin E, et al. Blue light induced A2E oxidation in rat eyes-experimental animal model of dry AMD. Photochem Photobiol Sci 2010;9:1505-12.

82. Tanito M, Agbaga MP, Anderson RE. Upregulation of thioredoxin system via Nrf2antioxidant responsive element pathway in adaptive-retinal neuroprotection in vivo and in vitro. Free radical biology \& medicine 2007;42:1838-50.

83. Green DG. Scotopic and photopic components of the rat electroetinogram. J Physiol 1973;228:781-97.

84. Buckser S. Spectral analysis of the albino rat electroretinogram. Curr Mod Biol 1968;2:1420.

85. Hardten DR, Noell WK. ERG of the albino rdy rat and susceptibility to light damage. Prog Clin Biol Res 1989;314:377-91.

86. Chader GJ, Taylor A. Preface: The aging eye: normal changes, age-related diseases, and sight-saving approaches. Investigative ophthalmology \& visual science 2013;54:ORSF1-4. 
87. Pinazo-Duran MD, Galbis-Estrada C, Pons-Vazquez S, Cantu-Dibildox J, Marco-Ramirez $\mathrm{C}$, Benitez-del-Castillo J. Effects of a nutraceutical formulation based on the combination of antioxidants and omega- 3 essential fatty acids in the expression of inflammation and immune response mediators in tears from patients with dry eye disorders. Clinical interventions in aging 2013;8:139-48.

88. Lee HS, Chauhan SK, Okanobo A, Nallasamy N, Dana R. Therapeutic efficacy of topical epigallocatechin gallate in murine dry eye. Cornea 2011;30:1465-72.

89. Chen W, Zhao K, Li X, Yoshitomi T. Keratoconjunctivitis sicca modifies epithelial stem cell proliferation kinetics in conjunctiva. Cornea 2007;26:1101-6. 